\title{
Is short course of antimicrobial therapy for asymptomatic bacteriuria before urologic surgical procedures sufficient?
}

\author{
Selda Sayin Kutlu ${ }^{1}$, Zafer Aybek ${ }^{2}$, Koray Tekin ${ }^{3}$, Demet Okke ${ }^{1}$, Serife Akalin ${ }^{1}$, \\ Serkan Altintas ${ }^{2}$, Melek Demir ${ }^{4}$
}

Departments of Infectious Diseases and Clinical Microbiology ${ }^{1}$, Urology ${ }^{2}$, General Surgery ${ }^{3}$, and Microbiology and Clinical Microbiology ${ }^{4}$, Pamukkale University, Denizli, Turkey

\begin{abstract}
Introduction: Duration of treatment of asymptomatic bacteriuria for patients undergoing urologic surgical procedures is undetermined. We compared the efficacy of long- versus short-course antimicrobial treatment in patients with asymptomatic bacteriuria undergoing urologic surgical procedures.

Methodology: Patients were divided into two groups according to duration of antimicrobial treatment. Group A patients received a single dose of an appropriate antibiotic, determined by antimicrobial sensitivity testing, 30 to 60 minutes before the surgical procedure. If a urinary catheter was placed postoperatively, a second dose was given following the recommended dose interval. Group B patients received antimicrobial treatment prior to surgery at least until patient urine became sterile. All patients were monitored for signs and symptoms of septicemia following surgical procedures.

Results: None of the patients enrolled in the study developed infectious complications such as sepsis or upper urinary tract infection. In group A, 31 patients were treated with antimicrobials before 39 urological procedures. In group B, the mean treatment time for 28 patients before 30 urological procedures was $8.03 \pm 3.86$ days. There were also significant differences in length of stay and the cost of antimicrobial therapy between the groups $(P<0.0001)$. Isolation of an increased number of resistant microorganisms was associated with long course therapy in group B.

Conclusions: Short course therapy protocol may be a practical, simple approach for antibiotic use; it decreases hospital stays, eliminates delayed procedure times, lowers the economic cost of antimicrobials and lessens the chance of superinfection with and spread of antimicrobial resistant microorganisms.
\end{abstract}

Key words: antimicrobial; bacteriuria; urologic surgical procedures

J Infect Dev Ctries 2012; 6(2):143-147.

(Received 15 December 2010 - Accepted 27 April 2011)

Copyright ( 92012 Sayin Kutlu et al. This is an open-access article distributed under the Creative Commons Attribution License, which permits unrestricted use, distribution, and reproduction in any medium, provided the original work is properly cited.

\section{Introduction}

Asymptomatic bacteriuria or asymptomatic urinary tract infections are common problems in particular groups such as catheterized patients while the catheter remains in situ, diabetic women, the elderly, and pregnant women [1]. Screening and treatment of asymptomatic bacteriuria in pregnant women and before some urological procedures has been recommended in the guidelines of the Infectious Diseases Society of America (IDSA) [2]. Preoperative bacteriuria was shown to increase the severe infectious complications such as bacteremia, septicemia, and upper urinary tract infection, especially following various urological interventions [3-6]. Accordingly, many studies have shown that appropriate antimicrobial treatment preoperatively and postoperatively could decrease the occurrence of these severe infectious complications [5,7,8]. However, the time interval that should be adhered to between antimicrobial therapy and the start of the surgical procedure as well as the length of the postoperative treatment is not clearly defined [1]. Long-term pre-treatment not only delays the operation, it may result in antibacterial resistance; however, a short course of antibiotic pre-treatment may not be sufficient to prevent postoperative bacteraemia [9]. The aim of the present study was to compare the effect of long-term versus short-course antimicrobial treatment in patients with asymptomatic bacteriuria undergoing specific urologic surgical procedures. 
Table 1. Characteristics of patients

\begin{tabular}{lccc}
\hline & Group A (n=31) & Group B (n=28) & $P$ value \\
\hline Age, year, mean (SD) & $59.43(18.13)$ & $58.97(15.72)$ & 0.917 \\
& & & \\
Male sex, $\mathrm{n}=43(\%)$ & $23(80.5)$ & $20(69)$ & 0.811 \\
Accompanying diseases (\%) & & & \\
$\quad$ Diabetes mellitus & $2(6.5)$ & $5(17.9)$ & 0.240 \\
Malignancy & $9(29.0)$ & $8(28.6)$ & 0.969 \\
Renal insufficiency & $6(19.4)$ & $4(14.3)$ & 0.434 \\
Previous hospitalization in the last 3 & & & \\
months (\%) & $16(41.0)^{*}$ & $21(70.0)^{\#}$ & 0.017 \\
Previous antibiotics in the last 3 months & & & \\
(except last 3 days) (\%) & $28(71.8)^{*}$ & $14(46.7)$ & 0.034 \\
Underlying conditions (\%) & & & \\
Benign prostate hyperplasia & $5(16.1)$ & $4(14.3)$ & 1.000 \\
Ureter stone & $3(9.7)$ & $8(28.6)$ & 0.095 \\
Renal stone & $8(25.8)$ & $4(14.3)$ & 0.342 \\
Prostate cancer & $3(9.7)$ & $3(10.7)$ & 1.000 \\
$\quad$ Bladder cancer & $4(12.9)$ & $3(10.7)$ & 1.000 \\
Urethral stenosis & $8(23.7)$ & $6(21.4)$ & \\
*39 procedures in group A \# 30 procedures in group B & &
\end{tabular}

\section{Methodology}

A total of 70 consecutive patients who had asymptomatic bacteriuria and undergone a number of urological surgical procedures between June 2005 and June 2008 were included in the study. Exclusion criteria were as follows: patients at risk of endocarditis, with prosthetic implants, or Serratia marcescens-related bacteriuria or neutropenia; patients who underwent solid organ or bone marrow transplantation; pregnant women; and patients below 18 years old. The patients were divided into two groups according to the duration of the antimicrobial treatment. Of the 59 patients, 31 were enrolled in a short course of antibiotic treatment (group A). A total of 39 procedures and antibiotic therapy were performed on these 31 patients. First clean-catch midstream urine specimens from patients with no symptoms of urinary tract infection were obtained 48 to 72 hours before the urological procedure. Antimicrobial susceptibilities were determined using the Kirby-Bauer disk diffusion method according to the Clinical and Laboratory Standards Institute (CLSI) published criteria [10]. According to antimicrobial sensitivity test results a single dose of appropriate parenteral antibiotic was given to the patients 30 to 60 minutes before the surgical procedure. This drug-to-procedure time interval was determined according to prophylaxis guidelines $[11,12]$. Following a dose interval recommended by the manufacturer, a second dose was given to patients with a postoperative urinary catheter. Urine samples were obtained immediately after the procedure for culture and antimicrobial sensitivity testing. All patients were followed clinically for the signs of upper urinary tract infections and sepsis. They were also informed about the signs and symptoms of infections and routine controls were performed for one week following discharge. The second group (group B) consisted of 28 patients who had been given long-term antimicrobial treatment (3 to 15 days) before and after a urologic surgical procedure between June 2005 and December 2006. A total of 30 procedures with antibiotic therapy were performed on these 28 patients. Passage of sterile urine cultures was confirmed in all of group B patients prior to the initiation of surgical procedures. Medical records of all patients in this group were evaluated for symptoms and signs of infections.

For asymptomatic women, bacteriuria was defined as two consecutive voided urine specimens with isolation of the same bacterial strain in quantitative counts $\geq 10^{5} \mathrm{cfu} / \mathrm{mL}$. For men bacteriuria was defined as a single, clean-catch voided urine specimen with one bacterial species isolated in a quantitative counts $\geq 10^{5} \mathrm{cfu} / \mathrm{mL}$. For either women or men, a single catheterized urine specimen with one bacterial species isolated in a quantitative count $\geq 10^{2}$ 
Table 2. Urologic procedures

\begin{tabular}{lcc}
\hline Urological prosedures & Group A (n=39) & Group B (n = 30) \\
\hline Transurethral prostatic resection (TUR-P) & 8 & 6 \\
Transuretral resection of bladder tumors (TUR-M) & 2 & 3 \\
Double J insertion and exchange & 12 & 11 \\
Cysostomy insertion & 5 & 2 \\
Nephrostomy tube insertion or exchange & 8 & 2 \\
Extracorporeal shock wawe lithotripsy (ESWL) & 1 & 1 \\
Ureterorenoscopy & 3 & 5 \\
\hline
\end{tabular}

$\mathrm{cfu} / \mathrm{mL}$ was also used to diagnose bacteriuria, according to the IDSA [2].

\section{Statistics}

Continuous variables were compared using Student's t test. Categorical variables were compared using the chi-square test. All significance tests were two sided at $\alpha<0.05$. All analyses were done with SPSS, version 13 (IBM, Chicago, IL, USA).

\section{Results}

During the study period, none of the patients enrolled in either group developed infectious complications such as sepsis or upper urinary tract infection. Demographic values and patient details regarding the associated pathologies and reasons for urologic procedures for both groups are shown in Table 1. A total of 69 urologic procedures were performed within both groups (Table 2). Distribution of the pathogens causing asymptomatic bacteriuria is shown in Table 3. Escherichia coli, Klebsiella pneumoniae, and Pseudomonas aeruginosa were the most frequently isolated microorganisms, respectively, in both groups. Because of the frequent occurrence of extended-spectrum beta-lactamase (ESBL) producing E. coli, $K$. pneumoniae and inducible beta-lactamase (IBL) producing $P$. aeruginosa at $60.5 \%(23 / 38), 83.3 \%(10 / 12)$ and $90 \%$ (9/10) respectively, imipenem was the most frequently selected antimicrobial agent used in both groups.

Post-procedural urine culture results showed that in group A, E. coli $(\mathrm{n}=4), K$. pneumoniae $(\mathrm{n}=1)$, and Candida tropicalis $(\mathrm{n}=1)$ were isolated from patients. These microorganisms were the same species and had the same antimicrobial sensitivity pattern to those isolated from pre-operative urine cultures with one exception. $C$. tropicalis was isolated from one post-operative urine culture while this patient had K. pneumoniae in pre-operative urine. While only a single dose of antimicrobial was administered pre-operatively in eight surgical procedures, a second dose was given post-operatively for 31 interventions due to the presence of a urinary catheter in group A patients. On the other hand, during the treatment period, we documented resistance to therapeutic antimicrobials in four patients from the long-term treatment group (group B). The mean treatment time was $8.03 \pm 3.86$ days in this group.

Pre-operative urinary catheterization was applied in $17(43.6 \%)$ procedures in group $\mathrm{A}$ and in 8 $(26.7 \%)$ procedures in group B. Mean hospitalization time was $1.82 \pm 1.43$ days in group A and $9.9 \pm 8.84$ days in group B $(P<0.0001)$. Mean antimicrobial cost was $31.84 \pm 1.06 \$$ in group $\mathrm{A}$ and $536.26 \pm$ $412.00 \$$ in group B $(P<0.0001)$. These differences were mainly due to long-term use of the antimicrobials.

\section{Discussion}

Results of our study showed that a short course of pre-operative antimicrobial therapy was apparently just as effective as long-term therapy in preventing severe infectious complications such as septicemia, and upper urinary tract infection following various urological procedures in patients with pre-operative asymptomatic bacteriuria. Long-term antimicrobial therapy was a common practice in our hospital until December 2006. However, in January 2007, we changed our protocol for management of asymptomatic bacteriuria before urological procedures to a short course of antimicrobial therapy as recommended by the IDSA guidelines [2]. Therefore, all patients treated with a short course of antimicrobial therapy from the beginning of January 2007 have been included prospectively in the present study.

Most of the randomized studies related to the prevention of post procedural infectious complications in asymptomatic bacteriuria have been done for transurethral prostatic resection (TUR-P); 
Table 3. Pathogens identified in urine

\begin{tabular}{lcc}
\hline & Group A $(\mathrm{n}=39)$ & Group B $(\mathrm{n}=30)$ \\
\hline E. coli & 21 & 17 \\
K. pneumoniae & 4 & 8 \\
P. aeroginosa & 7 & 3 \\
E. cloaca & 2 & 1 \\
Enterococcus spp & 1 & 1 \\
C. parapsilosis & 1 & 0 \\
C. tropicalis & 1 & 0 \\
A. baumanni & 2 & 0 \\
\hline
\end{tabular}

there are few studies documenting complications in asymptomatic bacteriuria for other procedures. Generally treatment of asymptomatic bacteriuria is recommended for any urological procedure in which mucosal bleeding is encountered $[2,5,13]$. In our study, along with TUR-P, other procedures were included.

Long-term treatment for bacteriuria prior to surgical procedures, to ensure that the urine is sterile before the procedure, can prevent severe infectious complications. However, long-term treatment may prolong the hospital stay, delay operation start time, increase hospitalization costs due to the increased use of antimicrobials, and may select for superinfection of the patient with more resistant organisms $[8,14,15]$. Similar to these reports, in our study the mean hospital stay and costs for antimicrobials were significantly higher in group B $(p<0.0001)$. Moreover, most probably due to the length of treatment, more resistant microorganisms were isolated from group B in our study. As a consequence of these potential problems, many researchers have recommended short-term antimicrobial therapy, the shortest of which starts immediately before the surgical procedure [8,13,16-19]. Interestingly, despite the efficacy of this preoperative short-term therapy, it has been shown that transient bacteraemia can occur with earlier initiation of antimicrobial therapy $[9,20]$. To determine the optimal time for starting preoperative antimicrobial therapy, Murphy et al. administered antibiotics at varying intervals before an operation. In this study, none of the patients whose treatment period exceeded 24 hours developed bacteraemia [9]. However, none of the patients who received appropriate antibiotics developed postoperative septicemia, whether or not bacteraemia had been observed during operations in the same study. On the other hand, Ohkawa et al. reported postoperative sepsis after prostatic surgery in five patients following an antimicrobial regimen which was administered one hour before and three hours after surgery, then subsequently twice daily. All five patients were bacteriemic as determined by blood culture, which was taken during or immediately after prostatectomy. In the same study, the authors recommended starting antimicrobials at least 12 to 24 hours pre-intervention [20]. Although bacteraemia was not evaluated in our study, we did not encounter any severe infectious complications such as sepsis postoperatively when administering antibiotics only 30 to 60 minutes before a surgical procedure.

Another consideration is the duration of the postoperative treatment. Duration of postoperative antibiotic treatment has been reported to range from 24 hours to 10 days [5-7,13,17,20-22]. A common recommendation in these studies is to continue antimicrobial therapy at least until removal of indwelling catheters. Interestingly, oral antimicrobials were continued during the postoperative period in one of the abovementioned studies although they were considered to have been unnecessary; the authors recommended assessment of effectiveness of a single antimicrobial dose preoperatively in future studies [17]. Accordingly, all group A patients in our study received a single preoperative, or if they had urinary catheter, a second parenteral, antimicrobial dose following the recommended dose interval postoperatively. As previously stated we did not encounter any severe infectious complications or septicemia postoperatively.

The treatment of antibiotic resistant microorganisms causing asymptomatic bacteriuria is more challenging and the observed frequency of 
resistant microorganisms, such as ESBL-producing $E$. coli and $K$. pneumoniae was notable. The soundest strategy would therefore be to prevent postoperative infectious complications, rather than attempted treatment of these resistant organisms.

\section{Conclusion}

In conclusion, we found that a single dose of parenteral antibiotic administered 30 to 60 minutes before the urologic procedure or a second dose of antimicrobial in cases where a urinary catheter remains in the patient, was as effective as long-term treatment, to prevent postoperative septicemia in a relatively small number of patients who have asymptomatic bacteriuria. We believe that this protocol is practical and easy to apply. Furthermore, it decreases the hospital stay, eliminates the delayed operation times, lowers the costs of antimicrobials, and more importantly lessens the chance of superinfection with resistant microorganisms.

\section{References}

1. Nicolle LE (2006) Asymptomatic bacteriuria: review and discussion of the IDSA guidelines. Int J Antimicrob Agents 28 Suppl 1: S42-48.

2. Nicolle LE, Bradley S, Colgan R, Rice JC, Schaeffer A, Hooton TM (2005) Infectious Diseases Society of America guidelines for the diagnosis and treatment of asymptomatic bacteriuria in adults. Clin Infect Dis 40: 643-654.

3. Grabe M (1984) Short antibiotic courses in transurethral prostatic resection. Scand J Urol Nephrol Suppl 78: 1-12.

4. Grabe M, Forsgren A, Bjork T (1986) Concentrations of ciprofloxacin in serum and prostatic tissue in patients undergoing transurethral resection. Eur J Clin Microbiol 5: 211-212.

5. Olsen JH, Friis-Moller A, Jensen SK, Korner B, Hvidt V (1983) Cefotaxime for prevention of infectious complications in bacteriuric men undergoing transurethral prostatic resection. A controlled comparison with methenamine. Scand J Urol Nephrol 17: 299-301.

6. Grabe M (1987) Antimicrobial agents in transurethral prostatic resection. J Urol 138: 245-252.

7. Grabe M, Forsgren A, Bjork T, Hellsten S (1987) Controlled trial of a short and a prolonged course with ciprofloxacin in patients undergoing transurethral prostatic surgery. Eur $\mathbf{J}$ Clin Microbiol 6: 11-17.

8. Grabe M, Forsgren A, Hellsten S (1984) The effect of a short antibiotic course in transurethral prostatic resection. Scand J Urol Nephrol 18: 37-42.

9. Murphy DM, Stassen L, Carr ME, Gillespie WA, Cafferkey MT, Falkiner FR (1984) Bacteraemia during prostatectomy and other transurethral operations: influence of timing of antibiotic administration. J Clin Pathol 37: 673-676.
10. Clinical and Laboratory Standarts Institute (2005) Performance Standards for Antimicrobial Susceptibility Testing. Clinical and Laboratory Standard Institute Wayne, Pennsylvania, 15 th Informational Supplement M7-A6.

11. Grabe M (2004) Controversies in antibiotic prophylaxis in urology. Int JAntimicrob Agents 23: S17-23.

12. Matsumoto T, Kiyota H, Matsukawa M, Yasuda M, Arakawa S, Monden K; Japanese Society of UTI Cooperative Study Group (Chairman: Tetsuro Matsumoto) (2007) Japanese guidelines for prevention of perioperative infections in urological field. Int J Urol 14: 890-909.

13. Hofer DR, Schaeffer AJ (1990) Use of antimicrobials for patients undergoing prostatectomy. Urol Clin North Am 17: 595-600.

14. Cafferkey MT, Falkiner FR, Gillespie WA, Murphy DM (1982) Antibiotics for the prevention of septicaemia in urology. J Antimicrob Chemother 9: 471-417.

15. Hall JC, Christiansen KJ, England P, Low AI, McRae PJ, Mander J, TaylerTA, Hall JL (1996) Antibiotic prophylaxis for patients undergoing transurethral resection of the prostate. Urology 47: 852-856.

16. Scherz HC, Parsons CL (1987) Prophylactic antibiotics in urology. Urol Clin North Am 14: 265-271.

17. Kiely EA, McCormack T, Cafferkey MT, Falkiner FR, Butler MR (1989) Study of appropriate antibiotic therapy in transurethral prostatectomy. Br J Urol 64: 61-65.

18. Grabe M, Forsgren A, Hellsten S (1986) The effectiveness of a short perioperative course with pivampicillin/pivmecillinam in transurethral prostatic resection: clinical results. Scand J Infect Dis 18: 567-573.

19. Kjaergaard B, Petersen E, Lauridsen KG, Petersen AS (1989) Prophylactic one-dose treatment with clindamycin and gentamicin in transurethral prostatic resection. A double-blind placebo controlled study. Scand J Urol Nephrol 23: 109-113.

20. Ohkawa M, Shimamura M, Tokunaga S, Nakashima T, Orito M (1993) Bacteremia resulting from prostatic surgery in patients with or without preoperative bacteriuria under perioperative antibiotic use. Chemotherapy 39: 140-146.

21. Grabe M, Forsgren A, Hellsten S (1984) A short antibiotic course given in conjunction with and after catheter removal consecutive to transurethral prostatic resection. Scand J Urol Nephrol 18: 193-199.

22. Charton M, Vallancien G, Veillon B, Prapotnich D, Mombet A, Brisset JM (1990) Use of antibiotics in the conjunction with extracorporeal lithotripsy. Eur Urol 17: 134-138.

\section{Corresponding author}

Selda Sayin Kutlu

Pamukkale University

Faculty of Medicine

Department of Infectious Diseases and Clinical Microbiology

Kinikli 20070 Denizli, Turkey

Telephone: +90-258-2118585 (2278)

Fax: 00902582410040

Email: sayinkutlu@yahoo.com

Conflict of interests: No conflict of interests is declared. 